\title{
Experiments in Moving Baseline Navigation using Autonomous Surface Craft
}

\author{
Joseph Curcio ${ }^{1}$, John Leonard ${ }^{1}$, Jerome Vaganay ${ }^{2}$, Andrew Patrikalakis ${ }^{1}$, \\ Alexander Bahr ${ }^{1}$, David Battle ${ }^{1}$, Henrik Schmidt ${ }^{1}$, and Matthew Grund ${ }^{3}$ \\ ${ }^{1}$ Massachusetts Institute of Technology, Cambridge, MA \\ ${ }^{2}$ Bluefin Robotics Corporation, Cambridge, MA \\ ${ }^{3}$ Woods Hole Oceanographic Institution, Woods Hole, MA
}

\begin{abstract}
This paper describes an on-going research effort to achieve real-time cooperative localization of multiple autonomous underwater vehicles. We describe a series of experiments that utilize autonomous surface craft (ASC), equiped with undersea acoustic modems, GPS, and $802.11 \mathrm{~b}$ wireless ethernet communications, to acquire data and develop software for cooperative localization of distributed vehicle networks. Our experiments demonstrate the capability of the Woods Hole acoustic modems to provide accurate round-trip and one-way range measurements, as well as data transfer, for a fully mobile network of vehicles in formation flight. Finally, we present preliminary results from initial experiments involving cooperative operation of an Odyssey III AUV and two ASCs, demonstrating ranging and data transfer from the ASCs to the Odyssey III.
\end{abstract}

\section{INTRODUCTION}

This paper describes a series of experiments using autonomous surface craft to develop and test distributed acoustic navigation algorithms for undersea vehicles. Our work is based on the concept of moving long baseline (MLBL) navigation [1], which was proposed by Vaganay and colleagues as a generalization of current long baseline (LBL) navigation to encompass mobile transponder networks. Whereas typical LBL navigation systems utilize pre-calibrated arrays of static transponders [2], [3], [4], in MLBL the goal is to create a self-calibrating, fully mobile network of autonomous underwater vehicles (AUVs) that can perform acoustic ranging and data exchange with one another to perform cooperative positioning.

Precision navigation is vital for the safety and effectiveness of all autonomous missions. Successful operation of an autonomous vehicle requires the ability to navigate. Navigation information consists of positional estimates and an understanding of the surrounding environment. Without this information, even the simplest of autonomous tasks are impossible. Improved navigation and coordination of multiple vehicles is essential to improve the accuracy and efficiency of mapping operations, such as mine-hunting, disaster response, and oceanographic surveys.

The idea of an underwater equivalent to the terrestrial Global Positioning System has long held appeal to AUV researchers. For example, A.C.S.A. has developed a portable undersea tracking range that provides a form of "underwater GPS" in a local area [5]. In the ACSA system, a network of four surface buoys equipped with GPS and RF communications utilize passive acoustic range measurements to track the position of a time-synchronized mobile undersea device. While the base system usually employs moored or drifted surface buoys, experiments using self-powered surface craft have also been performed. Further extensions of this concept could employ acoustic communications to relay the vehicle pose estimate obtained by the surface buoys back to the undersea vehicle itself.

The motivation behind our research is to enable multiple AUVs to cooperatively navigate. The ideal solution would enable heterogeneous teams of AUVs to operate with high navigational precision, without frequent surfacing for GPS measurements, even for the case when only a small number of the AUVs in the team are equiped with expensive international sensors. One application of this capability would be to perform rapid, large-area search with a mixed AUV network consisting of Comm/Nav-Aid AUVs, Search-Classify-Map AUVs, and Reacquire-Identify AUVs[1].

In previous work on MLBL, Vaganayet al. described an experiment utilizing two manned surface vessels as surrogate Comm/Nav-Aid vehicles and a single Odyssey III AUV, utilizing round-trip acoustic range measurements from Sonardyne's AvTrack acoustic navigation system. In this paper, we describe more recent experiments that have employed autonomous surface craft as Comm/Nav Aid vehicles and Woods Hole acoustic modems for ranging and for data transfer.

The problem of cooperative navigation of AUVs is highly interconnected to the problem of undersea acoustic communications [6], [7]. Our work capitalizes on recent progress by the Acoustic Modem Group at Woods Hole Oceanographic Institution in developing integrated communication and navigation (ranging) capabilities for small AUVs. Our application raises several interesting challenges for acoustic telemetry research, such as the need to handle a fully mobile network and the goal of achieving accurate one-way ranging through stable clock synchronization.

The objective of this paper is to provide an overview and progress report on a series of experiments in cooperative localization that have been performed over the past year. A novel feature of these experiments is that they utilize the MIT SCOUT (Surface Craft for Oceanographic and Undersea Testing) autonomous surface craft (ASC) as somobile platforms. While several other researchers have performed experiments with an individual ASC [8], [9], [10], we believe that ASCs offer extremely powerful capabilities when operated together in mobile vehicle networks. The use of an ASC network for cooperative 


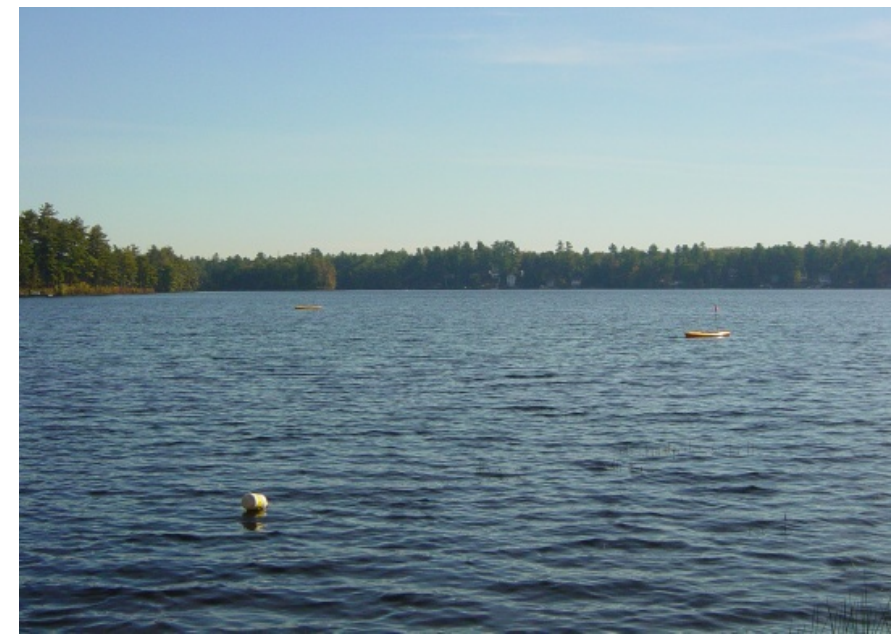

Fig. 1. Several Scout ASCs operating on Forest Lake for MLBL data acquisition trials.

AUV research is akin to using training wheels to ride a bike; GPS and WiFi communications greatly ease software development for tasks such as formation-keeping and cooperative position estimation. The cost, complexity, and risk of these experiments are at least an order of magnitude less than similar experiments would be with AUVs. GPS measurements also provide a convenient ground truth for the trajectory estimation process.

\section{FOREST LAKE 2004 EXPERIMENTS}

During September through November, 2004, a series of experiments were performed using three Scouts operated in Forest Lake, in Gray, Maine. Figure 1 shows several Scouts on the water for a typical experiment. A cooperative positioning behavior was written for the vehicles by adding an inter-vehicle communication process to the MOOS [11] robot operating software architecture. Changes to the formation-keeping algorithm configuration enabled rapid transition from follow-the-leader trajectories to formation-keeping (with a lead vehicle and two "wing-man" vehicles). Separation distances between the vehicles generally varied between 25 and 100 meters. A typical follow-the-leader trajectory is shown in Figure 2.

Figure 3 shows the manner in which the acoustic modems were mounted on each Scout - clearly a concern was that the close proximity of the transducer to the water surface would lead to degraded performance (especially given the shallow water in Forest Lake, which ranges for the most part between twenty and forty feet deep). Given these difficult conditions, however, the acoustic ranging performance was quite good, as shown in Figure 4, which illustrates the acoustic modem performance for these tests. More details of the modem configuration parameters, such as cycle times and data rates, will be published in an accompanying report. Figure 5 shows cooperative results obtained in post-processing from a typical mission, using a least-squares MLBL algorithm.

\section{AUVFEST 2005}

The next series of moving baseline navigation experiments involving MIT Scouts occurred during AUVFest 2005, two

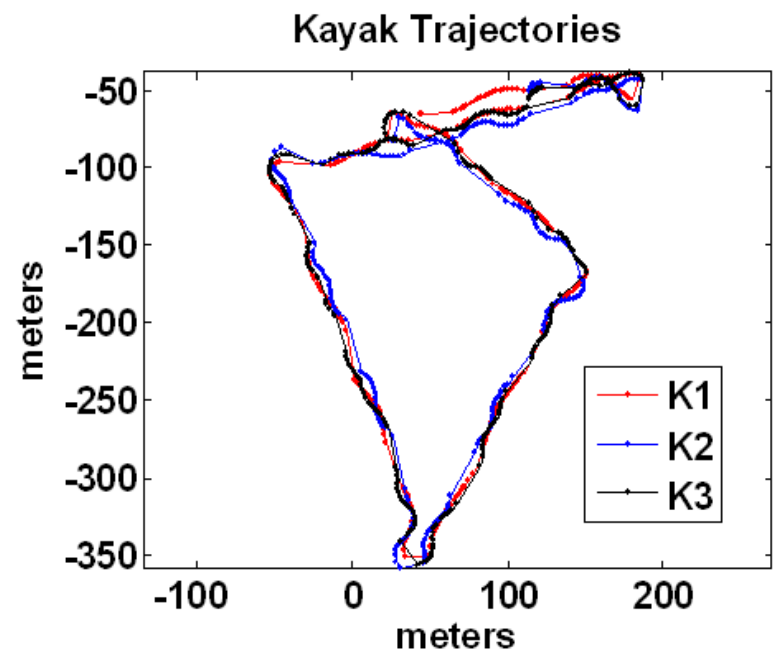

Fig. 2. Vehicle trajectories for a typical follow-the-leader missions.

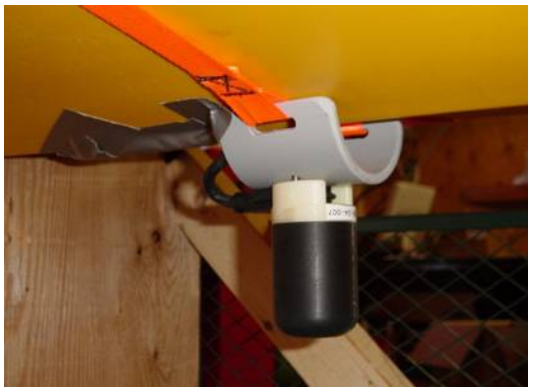

Fig. 3. Mounting of WHOI acoustic modem transducer on the Scout hull.

weeks of intensive AUV experimentation sponsored by the Office of Naval Research and held in Keyport, Washington in June 2005. The key technical advance for these experiments was the shift to using one-way acoustic range measurements with the WHOI modems, based on accurate time synchronization provided by GPS. We also experimented with different acoustic ranging configurations, alternating between transmission of short-packets for frequent ranging and longer packets encompassing data transfer. Figure 6(a) shows a photo of the four MIT Scouts deployed at AUVFest and Figure 6(b) shows the trajectories of three Scouts doing formation keeping and acoustic ranging during a typical experiment. Figures 6)(c) through Figures 6)(e) show a comparison of one-way acoustic range measurements with inter-vehicle distance measurements derived from GPS for this run. These data sets have been postprocessed with an MLBL algorithm to derive the trajectory of the lead vehicle in relation to the two following vehicle, as shown in Figure 6(f). Further algorithmic details will be published in a forthcoming report.

\section{Focused Acoustic Field 2005}

Most recently, we have performed initial experiments involving multiple Scouts and an Odyssey III AUV, during the NATO/ONE Focused Acoustic Field experiment in the Mediterranean in July, 2005. (See Figure 7.) Utilizing the precision time-synchronization of the Odyssey III's acoustic data acquisition system (developed by Eickstedt of MIT), we were able 


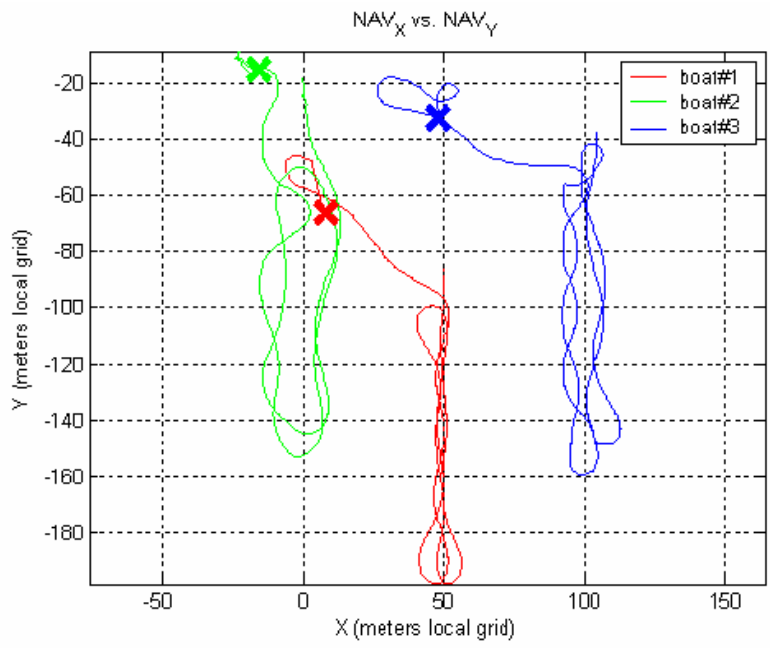

(a)

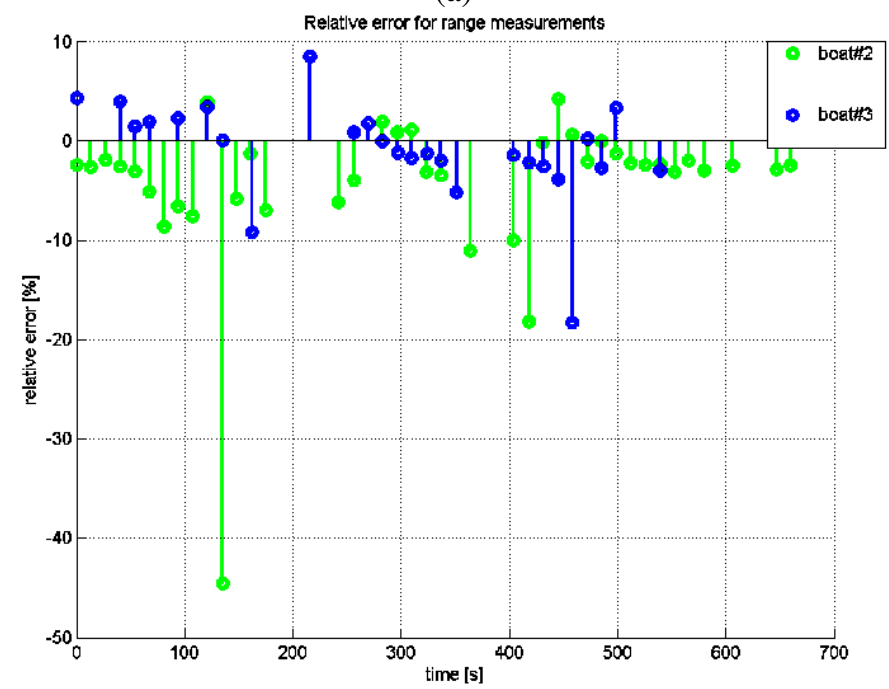

(b)

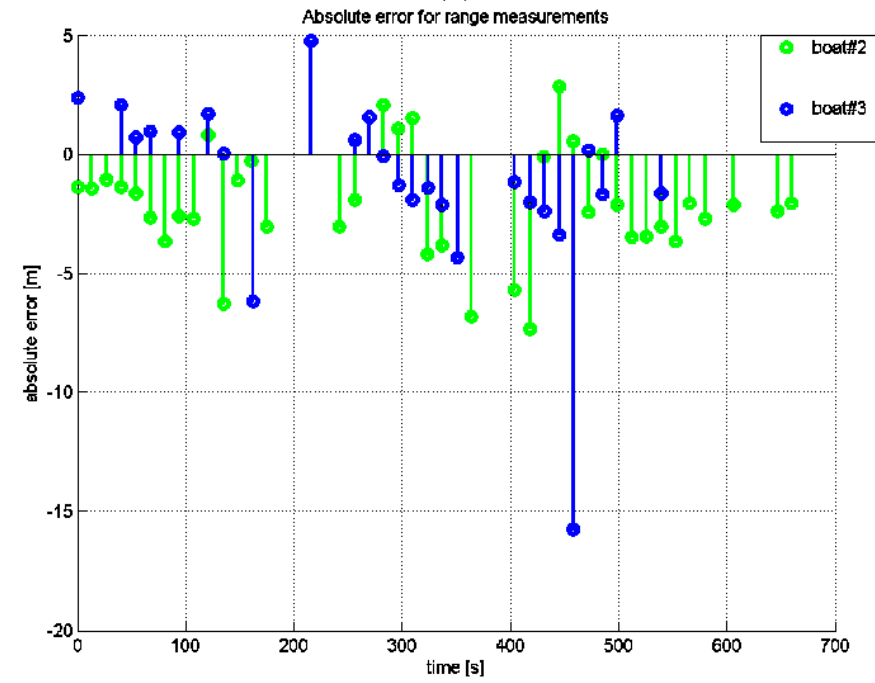

(c)

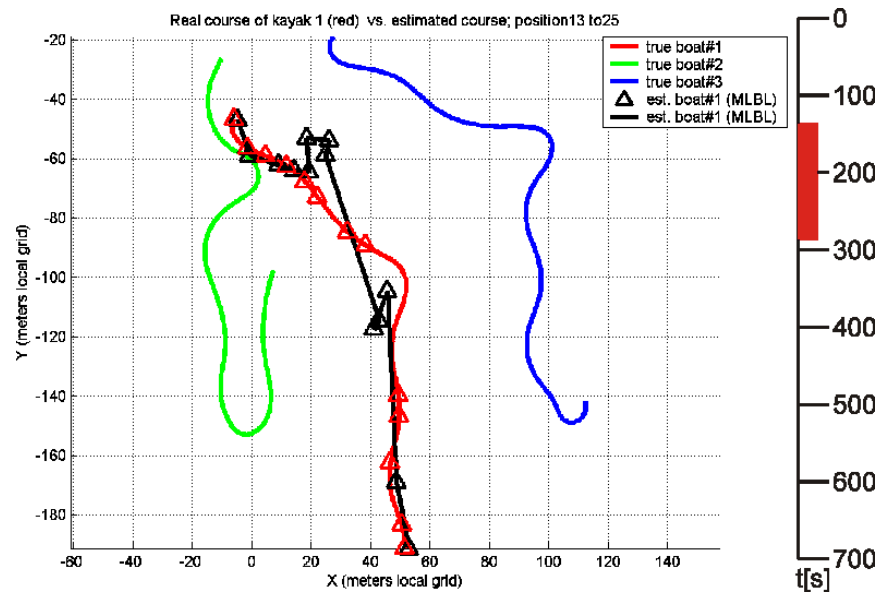

(d)

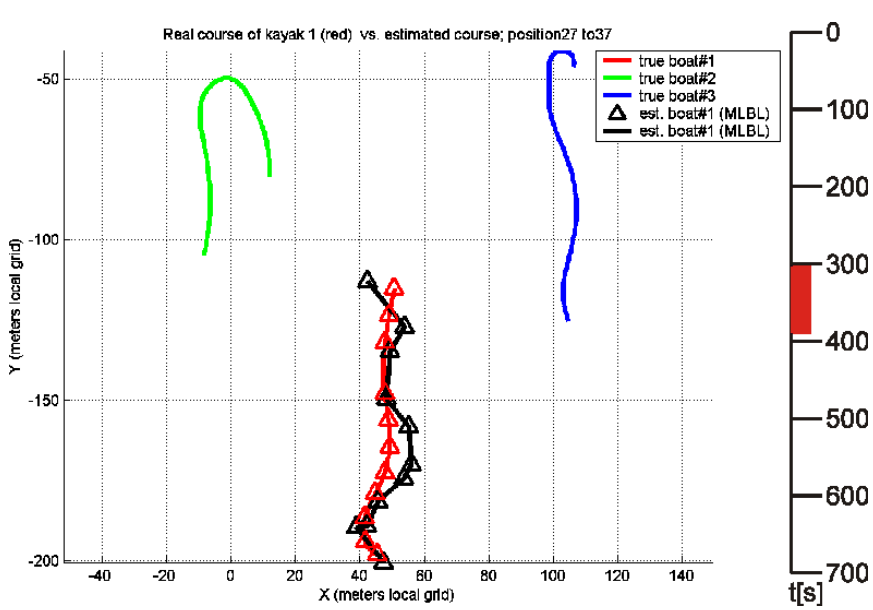

(e)

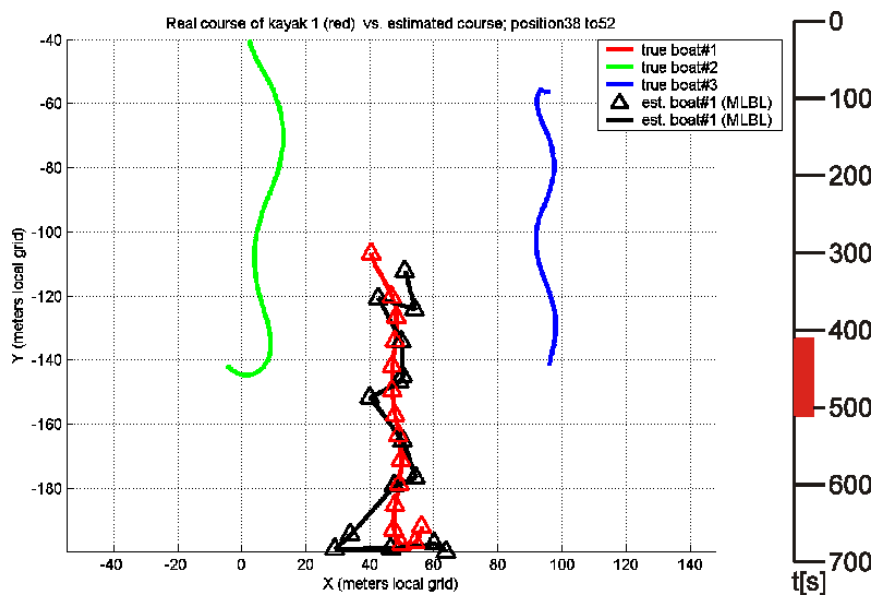

(f)

Fig. 5. Acoustic ranging results for Forest Lake 2004 experiment involving three kayaks. (a) vehicle trajectories (from GPS). (b) absolute range errors for round-trip acoustic ranging measurements. (c) relative range errors. (d)-(f) acoustically-derived vehicle trajectories for three different mission segments. 


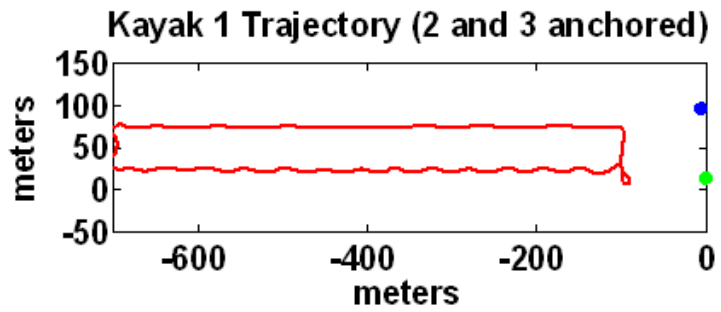

Distance between Kayak 1 and 3

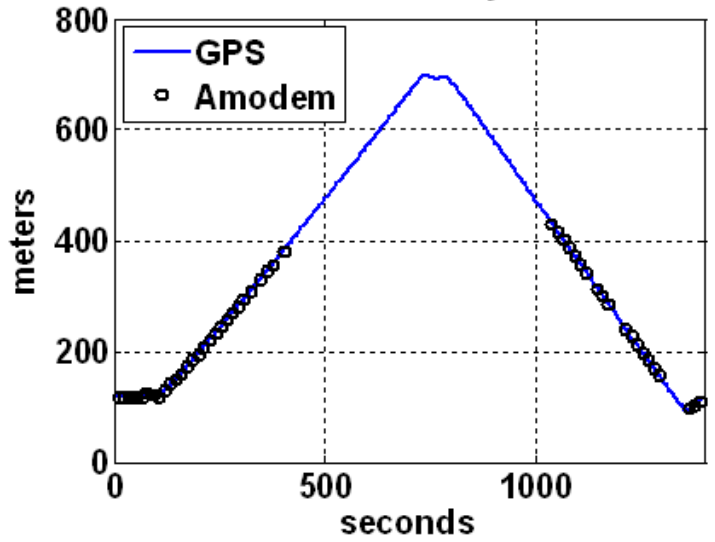

Distance between Kayak 1 and 2

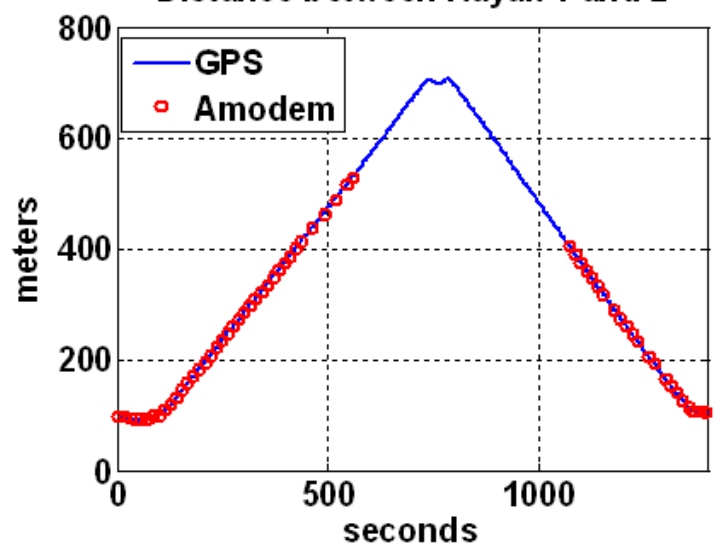

Range Diff between GPS and Amodem

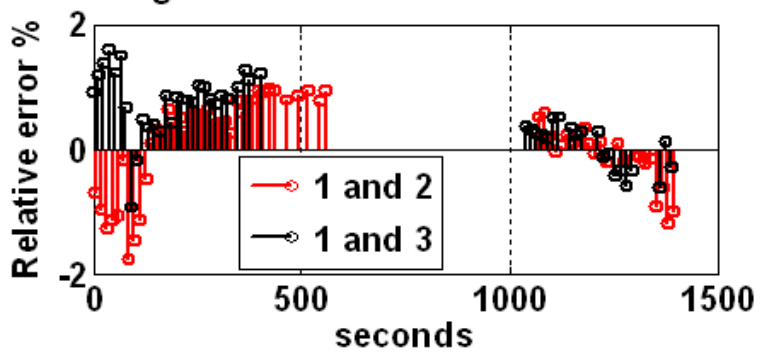

Fig. 4. Acoustic range measurement performance for Forest Lake 2004 experiment. (a) vehicle trajectory (from GPS) for a mission in which two Scouts were held stationary and a third Scout executed a long excursion to facilitate acoustic modem performance evaluation. (b)-(c) round-trip acoustic range measurements from the mobile kayak to each of the two stationary kayaks, as compared to GPS. Reliable ranging is indicated out to 400 meters range, which exceeded our performance expectations given the very shallow water in the Lake and the mounting position of the transducer so close to the kayak hull. (d) range errors for round-trip acoustic ranging measurements, expressed as relative percentage error. to obtain one-way acoustic ranging measurements between two Scouts and an Odyssey III AUV for the first time. During one day of joint operations, a total of 337 time-synchronized data packets transmitted from the Scout vehicles were successfully received by the Odyssey III AUV. Preliminary results indicate some difficulties with reception of data packets while the vehicle was in motion, which was likely due to vehicle self-noise and a challenging acoustic environment. Work in progress is processing and analyze these data sets, and will be published in a future report. Additional experiments are also being planned.

\section{CONCLUSION}

This paper has described a series of experiments system for development of algorithms to enable real-time cooperative localization for multiple AUVs. The experiments have utilized MIT Scout autonomous surface craft equiped with acoustic modems, GPS, and 802.11b wireless ethernet communications to acquire moving baseline data and to test cooperative adaptive behavior algorithms. Preliminary results have been reported, demonstrating the capability to utilize either round-trip or oneway acoustic range measurements to perform cooperative position estimation. Numerous lessons learned with these systems are expected to pay-off in improved effectiveness of multiple AUV experiments planned for the near future.

\section{ACKNOWLEDGMENTS}

This work was supported by ONR Grant N00014-02-C-0210, part of the Autonomous Operations Future Naval Capabilities program (Sponsor: J. Valentine), and by the ONR Undersea Persistent Surveillance program. The authors are grateful to numerous staff at MIT, Bluefin, and the Woods Hole Oceanographic Institution whose efforts made these experiments possible, especially Scott Willcox, Lee Freitag, Keenan Ball, Don Eickstedt, Jacques Leederkerken, Mike Benjamin, Matthew Walter, and Robert Williams.

\section{REFERENCES}

[1] J. Vaganay, J. Leonard, J. Curcio, and S. Willcox, "AOFNC - experimental validation of the moving long base line navigation concept," in $A U V$ 2004, June 2004.

[2] P. H. Milne, Underwater Acoustic Positioning Systems. London: E. F. N. Spon, 1983.

[3] J. Vaganay, J. G. Bellingham, and J. J. Leonard, "Outlier rejection for autonomous acoustic navigation," in Proc. IEEE Int. Conf. Robotics and Automation, April 1996, pp. 2174-2181.

[4] L. Whitcomb, D. Yoerger, H. Singh, and J. Howland, "Advances in underwater robot vehicles for deep ocean exploration: navigation, control, and survey operations," in Robotics Research: The Ninth International Symposium, D. Koditschek and J. Hollerbach, Eds. Snowbird, Utah: Springer Verlag, 2000.

[5] H. Thomas, "Commercial offer: Gps intelligent buoy system (a.c.s.a., http: / / www . underwater-gps . com)," 2001.

[6] J. Catipovic, "Performance limitations in underwater acoustic telemetry," IEEE J. Ocean Engineering, vol. 15, no. 3, pp. 205-216, July 1990.

[7] D. B. Kilfoyle and A. B. Baggeroer, "The current state-of-the-art in underwater acoustic telemetry," IEEE J. Ocean Engineering, vol. 25, no. 1, pp. 4-27, 2000.

[8] T. Vaneck, C. Rodriguez-Ortiz, M. Schmidt, and J. Manley, "Automated bathymetry using an autonomous surface craft," Navigation: Journal of the Institute of Navigation, vol. 43, no. 4, 1996.

[9] J. E. Manley, "Development of the autonomous surface craft ACES," in IEEE Oceans, 1997.

[10] J. E. Manley, A. Marsh, W. Cornforth, and Wiseman, "Evolution of the autonomous surface craft autocat," in IEEE Oceans, 2000.

[11] P. Newman, "MOOS - a mission oriented operating suite," Department of Ocean Engineering, Massachusetts Institute of Technology, Tech. Rep., 2002. 


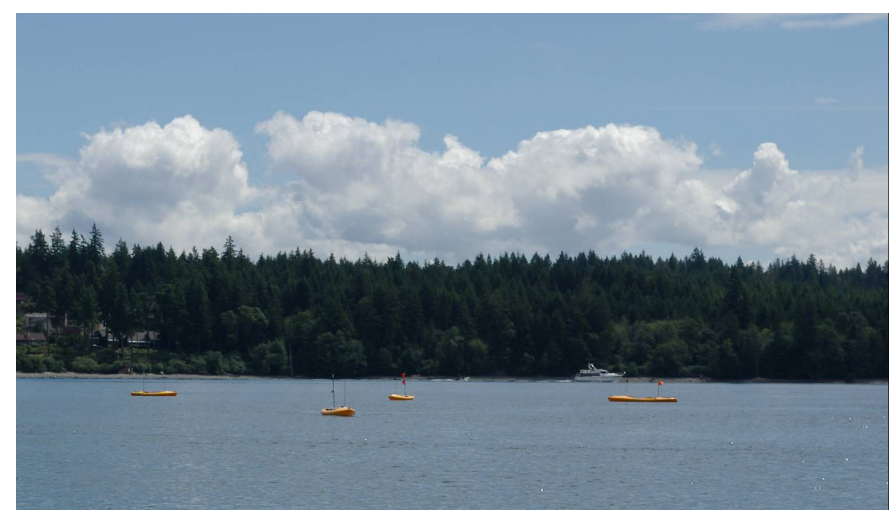

(a)

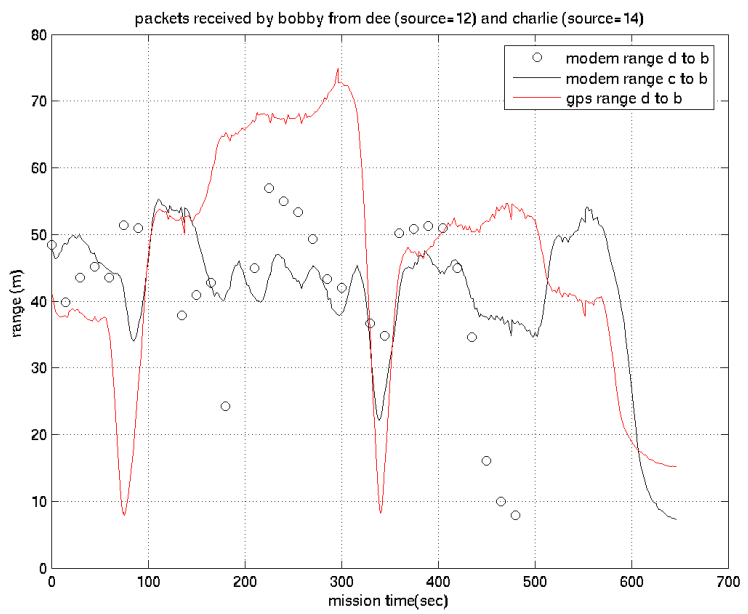

(c)

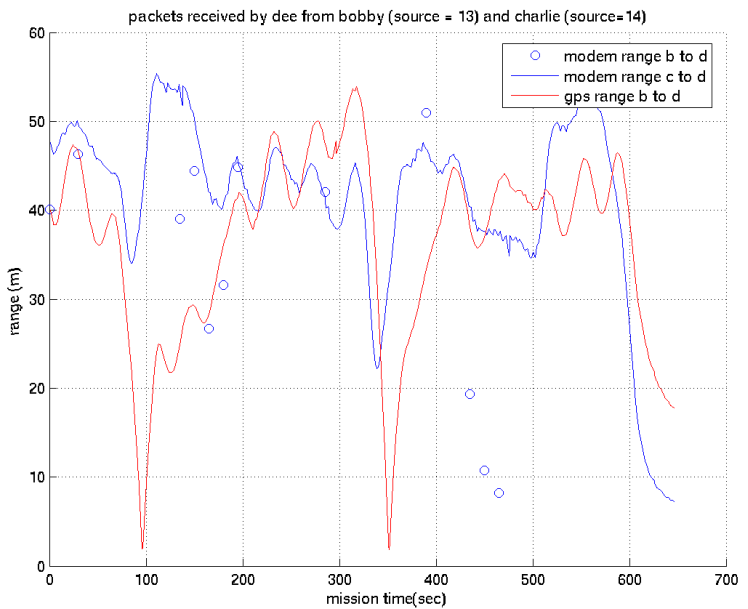

(e)

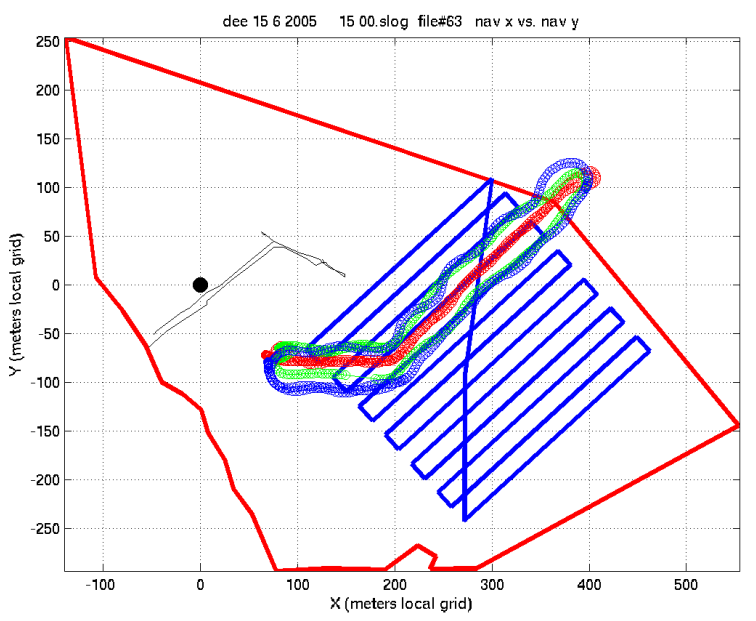

(b)

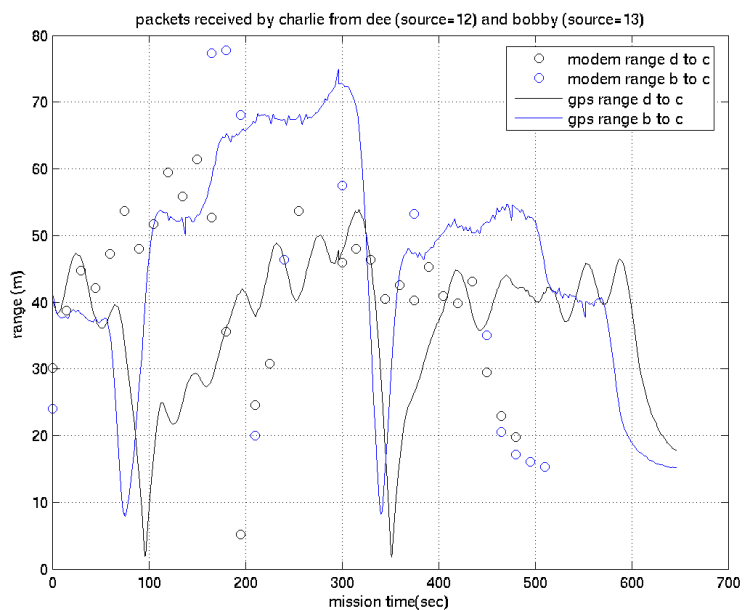

(d)

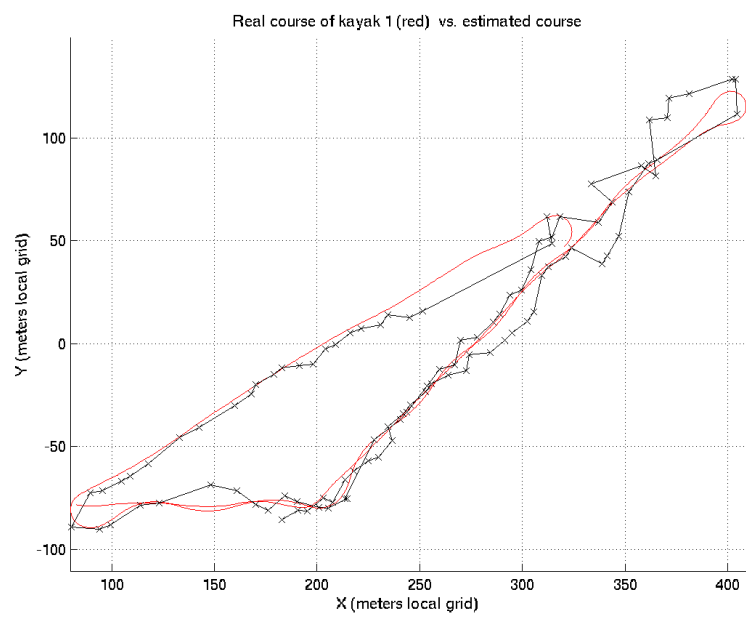

(f)

Fig. 6. One-way Acoustic ranging results for three kayaks performing cooperative formation-keeping missions at AUVFest 2005 in Keyport, Washington. (a) MIT Scout AUVs performing cooperative formation keeping and acoustic ranging at AUVFest in Keyport, WA, in June 2005. (b) vehicle trajectories (from GPS). (c)-(e) absolute range errors for one-way acoustic ranging measurements for the three Scouts (Bobby, Charlie, and Dee). (f) MLBL computed vehicle trajectory for the lead kayak using GPS positions for the other two vehicles and one-way acoustic ranges. 


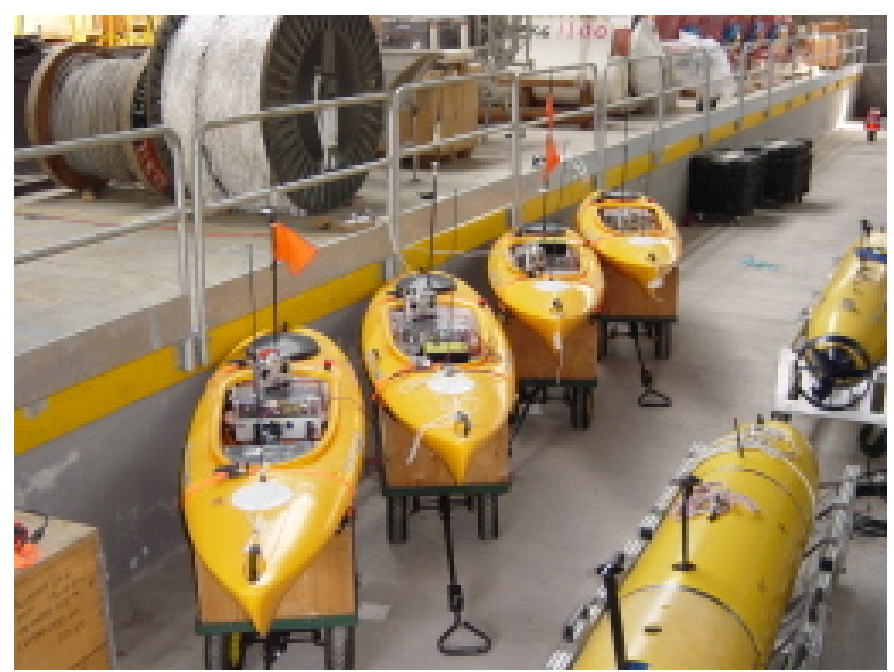

(a)

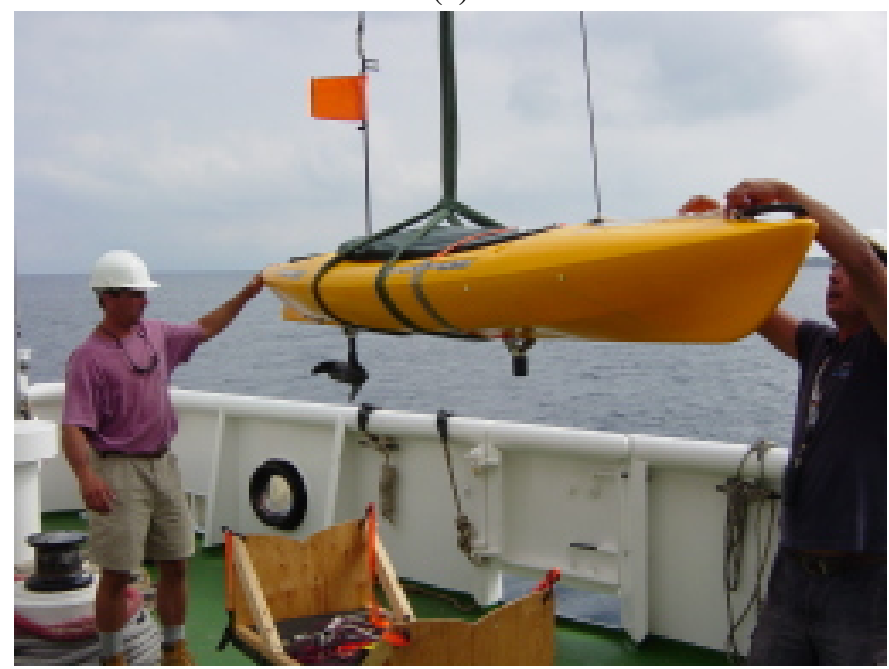

(c)

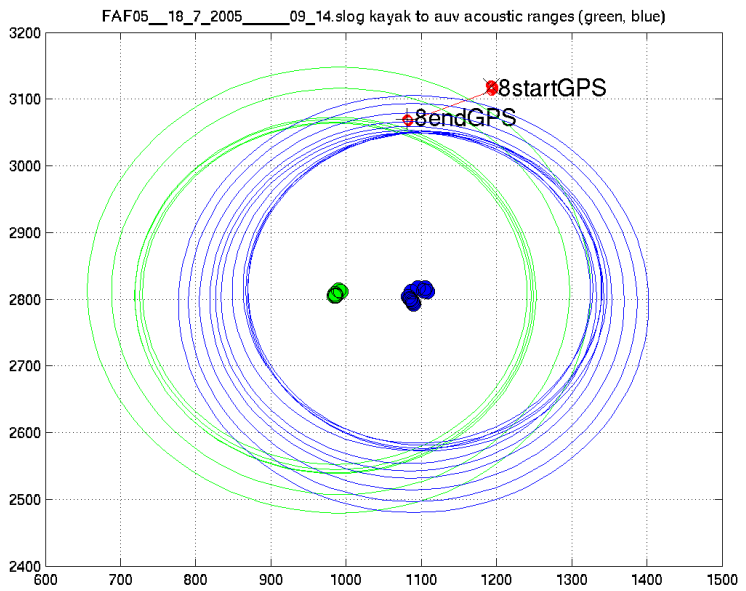

(e)

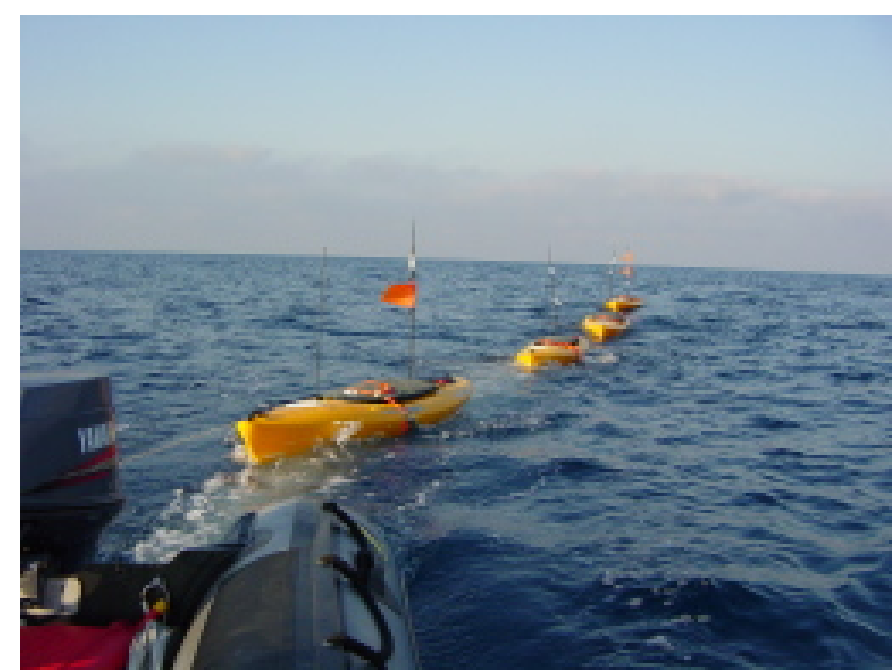

(b)

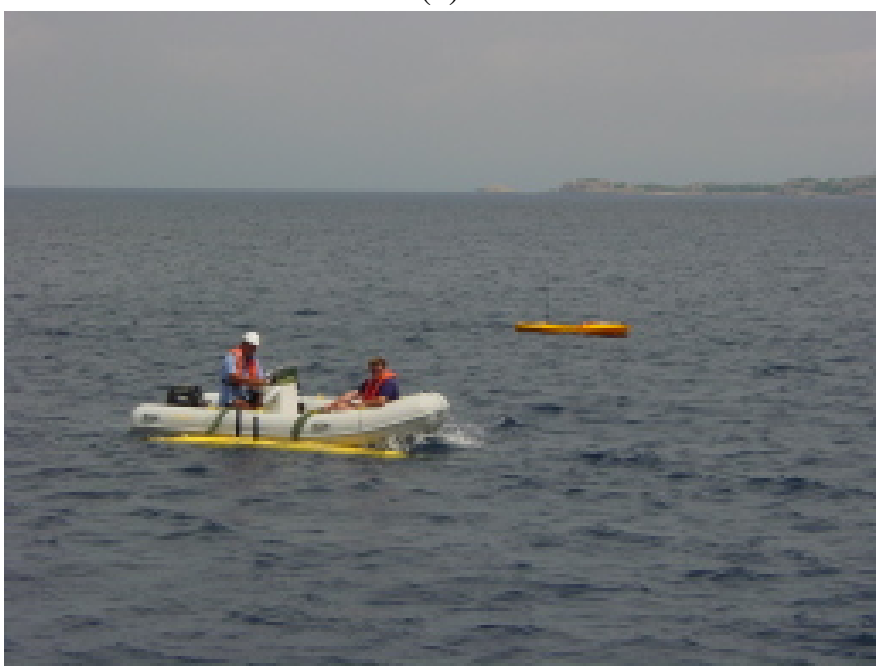

(d)

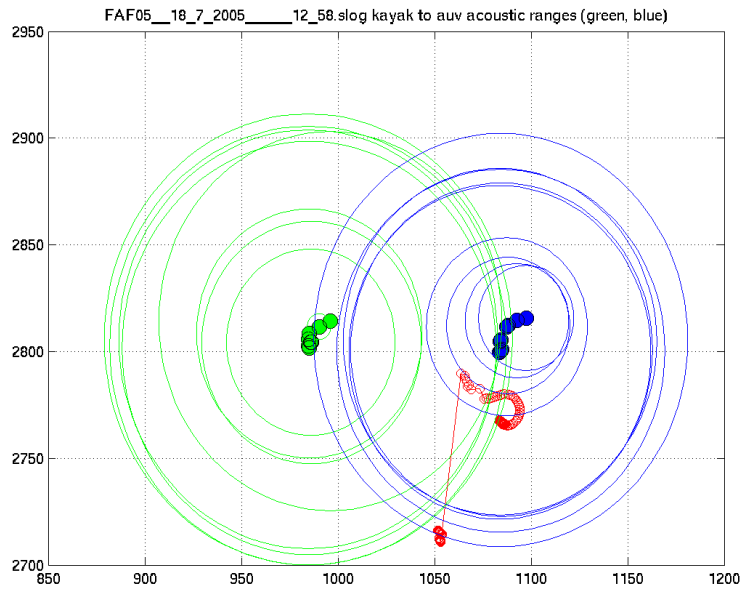

(f)

Fig. 7. Deployment of four MIT Scout ASCs and two Odyssey IIIs at the Focused Acoustic Field (FAF) 2005 experiment in Italy. (a) Four Scouts and two Odyssey IIIs after shipment. (b) Four Scouts in tow to operations site at which four-AUV one-way-ranging and data transfer experiments were performed. (c) A Scout vehicle being launched from NATO R/V LEONARDO. (d) The Odyssey III AUV Unicorn and one of the MIT Scouts during joint AUV/ASC operations at FAF-05. (e)-(f) One-way acoustic range measurements displayed as circles referenced to the center of each of the Scout vehicles (blue and green circles) for two short missions (two minutes and six minutes respectively). GPS measurements of the AUV position at the start and end of the mission are shown as red circles. 